\title{
Health Education Policies to Maximize the Effectiveness of Human Resources for Health Programs in Low-Income Countries
}

\author{
Cameron Page, $\mathrm{MD}^{1,2}$ and Vincent Sugira, $\mathrm{MMED}^{1,3}$ \\ 'Department of Internal Medicine, College of Medicine and Health Sciences, University of Rwanda, Kigali, Rwanda; ${ }^{2}$, Brooklyn, NY, USA; ${ }^{3}$ Rwanda \\ Military Hospital, Kigali, Rwanda.
}

$\mathrm{J}$ Gen Intern Med 32(5):497-9

DOI: $10.1007 / \mathrm{s} 11606-016-3934-5$

(c) Society of General Internal Medicine 2016

\section{INTRODUCTION}

In July 2012, the President of Rwanda, Paul Kagame, launched the Human Resources for Health Program (HRH). Speaking at the event, former President Bill Clinton called HRH "the largest medical education project ever undertaken," one that would "pave the way for a new paradigm in foreign aid and global health partnerships." ${ }^{1}$ Funded in part by the United States through the President's Emergency Plan For AIDS Relief (PEPFAR), and in part by the UN Global Fund, the Rwandan HRH program partnered with U.S. universities to bring physicians, nurses, dentists, and midwives to Rwanda to teach. ${ }^{2}$ The internal medicine residency at the University of Rwanda has been augmented by U.S. internists, who come to the country to teach and stay for 12 months or more. Subspecialists within internal medicine stay for 3 to 6 months at a time. There have been a number of articles published about the operational details of the Rwandan HRH program, and efforts are currently under way to expand the HRH model to other low-income countries.

There is widespread agreement that educating the next generation of doctors and nurses is essential. In the setting of limited resources, however, difficult choices must sometimes be made between medical education and clinical care. We have observed that in these situations, education often suffers, for obvious reasons: the benefit of a polio vaccination program is clear and measurable, but the value of teaching and learning is less quantifiable.

The tension between education and care delivery can be relieved somewhat through a large-scale capacity building program like HRH. The presence of visiting faculty means that clinical services do not have to be reduced in order to boost education. But HRH is a temporary initiative; to maxi-

Received May 2, 2016

Revised August 10, 2016

Accepted November 15, 2016

Published online December 5, 2016 mize the program's impact, all health education policies should be evaluated and revised prior to commencement.

Health education policy considerations fall into three broad categories: medical students, post-graduates, and faculty.

\section{MEDICAL STUDENTS}

All Rwandan medical school graduates work in a rural district hospital for 2 years after graduation. They work as general practitioners (GPs) and have limited interactions with senior professional colleagues or mentors. After their service requirement, GPs can apply to sub-specialty training programs, including internal medicine. Some Rwandan physicians, however, for personal or financial reasons, continue to work as GPs for many more years before applying for subspecialization.

This contrasts with the United States, which has no service requirement. Learners move seamlessly between medical school and residency, two environments that are both academic and supervised. The good habits that are inculcated during medical school have no opportunity to atrophy before residency: independent thought, proactive self-education, appropriate skepticism of a received diagnosis, and critical appraisal of the medical literature.

In Rwanda, the doctor-to-population ratio is 6 per 100,000 (in the United States it is 250 , and in urban areas 375 ). ${ }^{3}$ In the setting of physician scarcity, it is completely reasonable for the Rwandan government to maximize the clinical responsibilities of new medical school graduates. While this policy may be correct from a health policy perspective, however, it introduces challenges for the internal medicine faculty when these doctors return for residency training. Many internal medicine residents, although smart, hard-working, and dedicated to their patients, have developed bad habits (or simply outdated ones) during their stints as GPs. A general shift toward a less hierarchical and more Socratic educational system in Rwanda would also be beneficial.

The Rwandan Ministry of Health allows a few of the highest-performing medical students to skip the 2-year service requirement and move immediately to specialization. This policy should be considered by other low-income countries that adopt an HRH model. 


\section{Post-Graduates}

Once new internal medicine specialists are trained, there is strong pressure to move these physicians away from the academic centers toward the district hospitals, where they can use their skills to manage complex patients. This dissemination of internists into rural areas is an excellent and appropriate goal. As with the placement of medical school graduates, however, a balance must be struck.

Each year, the academic medical centers retain a few newly graduated internists to serve as junior faculty. In Rwanda, this practice is being expanded as the HRH program moves toward its conclusion. Instead of placing all new internal medicine specialists in rural district hospitals, the Ministry of Health is retaining a higher proportion in the academic medical centers, giving preference to those who show the most aptitude for teaching. One attractive feature of this proposal is that it is revenue-neutral, requiring only minor adjustments in human resource allocation.

As HRH ends, if visiting U.S. physicians are not replaced by Rwandan doctors, the number of teachers will drop and the quality of the education will decline. Gains made in the number of internal medicine doctors in Rwanda might be reversed. Furthermore, if the quality of teaching in the residency drops, then the reputation of internal medicine as a specialty will go down, which will weaken the value of an internal medicine specialization and hamper recruitment.

HRH is a short-term, time-limited program, which brings added educational resources. It is only logical for the host country to expose as many students, postgraduates, and junior faculty to these HRH visitors as possible. Keeping a high number of residency graduates in the academic teaching centers for the duration of an HRH program is one of the best ways to maximize the effectiveness of HRH.

We understand and agree with the argument that the purpose of training internal medicine doctors is to allow them to serve the citizens of Rwanda. The country is at a critical juncture, however, in the development of its healthcare workforce. If there is strong investment in the field of internal medicine over the next 5-10 years, the quality of the healthcare system can leapfrog ahead of neighboring countries. A decade allows for several generations of postgraduates to complete residency training and join the junior faculty, but is not so long that it prevents the government from working toward larger health system goals.

Allocating more internists to teaching duties might mean that it takes slightly longer to disseminate internal medicine specialists to the most rural areas. But steady progress toward a goal is better than rapid gains follow- ed by reversal. Although there is no indication that Rwanda is on track for any reversal in the impressive health gains that have been posted so far, ${ }^{4,5}$ a balance should be struck between increasing these gains and maintaining health system sustainability for future decades.

\section{Faculty}

Visiting HRH faculty have the luxury of dedicating themselves to teaching, but Rwandan faculty members in the internal medicine department are tasked with significant clinical duties. As HRH ends and visiting faculty leave, there is a risk that Rwandan faculty will have to return to their clinical work, and education will suffer.

If internal medicine teaching is appropriately valued, then the residency program will continue to grow and improve. If the Rwandan healthcare system cannot find a way to place a high value on education, however, then the impressive advances posted so far cannot be sustained.

How can top-quality teaching be encouraged? The most important factor is the existence of flagship academic medical centers where doctors are proud to work. Currently, some doctors see teaching as an added burden, unappreciated by their peers and superiors. The job of clinician educator must be reframed as a privilege: of all the physicians in the country, only a handful are given the honor of shaping the next generation of internists.

In conversations with residents who underwent training during the time of $\mathrm{HRH}$, many of them mentioned the topic of "institutional culture." There were always some excellent Rwandan professors, but the addition of HRH faculty established a critical mass of doctors committed to evidencebased academic medicine. This allowed a culture change to begin, toward consistent excellence and the expectation of regular high-quality teaching. While there are many health problems in low-income countries that outside doctors cannot solve, one way they can clearly contribute is by supporting local faculty in their efforts to build a culture of excellence.

It is also appropriate to materially reward top-quality teaching. This prompts the question: who decides which teachers are the best? In Rwanda, as in many places in the United States, there is currently no rigorous quantitative system of teacher evaluation whose results are generally accepted as accurate. Without an open, transparent teacher evaluation process, any incentives or perquisites given to top teachers may be perceived as unfair, regardless of whether they actually are. The design, validation, and implementation of a teacher evaluation process would be a suitable project for funding by a private donor, either individual or institutional. 


\section{CONCLUSIONS}

Building and sustaining an internal medicine residency in a low-income country can be a challenge, and HRH can play a useful role. Prior to commencing a large-scale HRH program, low-income countries should revise their health education policies, with a focus on maximizing the educational impact of the HRH initiative.

Acknowledgments: We wish to thank Dr. Beth Riviello, MFD, MPH, for her input on this manuscript. We received no grants or financial support for this work.

The views expressed in this article are solely those of the authors, and do not represent an official position of the University of Rwanda or the Rwandan Military Hospital.

Corresponding Author: Cameron Page, MD; Department of Internal Medicine, College of Medicine and Health SciencesUniversity of Rwanda, KN 4th Avenue, Kiyovu, Kigali, Rwanda (e-mail: cameron. page@gmail.com).

\section{Compliance with Ethical Standards:}

Conflict of Interest: The authors declare that they do not have a conflict of interest.

\section{REFERENCES}

1. Musoni E. Bill Clinton Announces New Medical Assistance to Rwanda The Rwanda New Times [Internet]. 2012 Jul 20; Available from: http:// www.newtimes.co.rw/section/article/2012-07-20/55263/. Accessed November 2, 2016.

2. Nuthulaganti T, Umubyeyi B, Sc MN, Nyemazi JP, Uwayezu A, Rusanganwa V, et al. The Human Resources for Health Program in Rwanda-A New Partnership. N Engl J Med. 2013;369(2 1):2054-8.

3. CIA World Factbook. 2012.

4. Farmer PE, Nutt CT, Wagner CM, Sekabaraga C, Nuthulaganti T, Weigel JL, et al. Reduced premature mortality in Rwanda: lessons from success. BMJ Open. 2013;346(1):f65-f65.

5. Binagwaho A. Health Sector Annual Report. Rwanda Ministry of Health [Internet]. Available from: http://www.moh.gov.rw/fileadmin/templates/ MOH-Reports/HEALTH_SECTOR_ANNUAL_REPORT_July_2013-June 2014.pdf. Accessed November 2, 2016. 